\title{
Note on the Computation of the Bessel Function $I_{\mathbf{n}}(\mathbf{x})$.
}

The Bessel function of purely imaginary argument

$$
I_{n}(x)=i^{-n} J_{n}(i x)=\sum_{m=0}^{\infty} \frac{(x / 2)^{n+2 m}}{\Gamma(m+1) \Gamma(m+n+1)}
$$

is of frequent occurrence in applied mathematics, statistics, and asymptotic number theory. The calculation of $I_{n}(x)$ for $n$ and $x$ both large presents an interesting problem to the computer. ${ }^{1}$ It is clear that the series for $I_{n}(x)$, (though everywhere convergent) is of little practical use for large $x$. Even the well-known asymptotic formula, designed for use with large $x$

$$
\text { (2) } I_{n}(x)=\frac{e^{x}}{(2 \pi x)^{\frac{1}{2}}}\left(1-\frac{4 n^{2}-1^{2}}{1 ! 8 x}+\frac{\left(4 n^{2}-1^{2}\right)\left(4 n^{2}-3^{2}\right)}{2 !(8 x)^{2}}-\cdots\right)
$$

fails to be of practical use when $n$ is of order $\sqrt{x}$.

Bessel functions of large integral order can, theoretically, be built up from the values of $I_{0}(x)$ and $I_{1}(x)$ (given in tables, ${ }^{2}$ or found by (2)) by use of the recurrence formula

$$
I_{k+1}(x)=-2 k I_{k}(x) / x+I_{k-1}(x) .
$$

This procedure, however, affords another example in which great accuracy is needed to start with (as has been pointed out in case of $J_{n}(x)$ by Cosens [MTAC p. 99]), since more and more accuracy is lost at each step. The value of $I_{90}(60)=0.0725775 \cdots$ would, if computed this way, require about 40 decimal places in $I_{0}(60)$ and $I_{1}(60)$, and 89 applications of (3).

Nicholson ${ }^{3}$ has given a formula for $I_{n}(x)$ purporting to be "suitable for rapid tabulation," when either $n$ or $x$ is large. The formula is not given explicitly (except its first term) but in terms of an infinite differential operator with undetermined coefficients operating on a cumbersome function of $n$ and $x$.

The following formula, which I have not found in the literature, is quite effective in case $n$ is large and $x$ has any real value.

$$
I_{n}(n x)=\frac{1}{(2 \pi n z)^{\frac{1}{3}}}\left\{(z-1) e^{z} / x\right\}^{n} \exp \sum_{m=1} \psi_{m}\left(x^{2}\right) /\left(n z^{3}\right)^{m}
$$

where $z=\left(1+x^{2}\right)^{\frac{1}{2}}$, and where the $\psi$ 's are the following polynomials:

$$
\begin{aligned}
24 \psi_{1}\left(x^{2}\right)= & 3 x^{2}-2, \\
16 \psi_{2}\left(x^{2}\right)= & x^{4}-4 x^{2}, \\
5760 \psi_{3}\left(x^{2}\right)= & 375 x^{6}-3654 x^{4}+1512 x^{2}-16, \\
128 \psi_{4}\left(x^{2}\right)= & 13 x^{8}-232 x^{6}+288 x^{4}-32 x^{2} \\
322560 \psi_{5}\left(x^{2}\right)= & 67599 x^{10}-1914210 x^{8}+4744640 x^{6}-1891200 x^{4} \\
192 \psi_{6}\left(x^{2}\right)= & 103 x^{12}-4242 x^{10}+17493 x^{8}-14884 x^{6}+2580 x^{4}-48 x^{2}, \\
3440640 \psi_{7}\left(x^{2}\right)= & 5635995 x^{14}-318291750 x^{12}+1965889800 x^{10} \\
& \quad-2884531440 x^{8}+1135145088 x^{6}-99783936 x^{4} \\
4096 \psi_{8}\left(x^{2}\right)= & 23797 x^{16}-1765936 x^{14}+15252048 x^{12}-34280896 x^{10} \\
& \quad+24059968 x^{8}-5095936 x^{6}+248320 x^{4}-1024 x^{2} .
\end{aligned}
$$


This formula may be derived from an asymptotic expansion of the solution

of the differential equation

$$
y=d\left(\ln I_{n}(n x)\right) / d x
$$

$$
y^{\prime}+y / x+y^{2}=n^{2}\left(1+x^{2}\right) / x^{2}=(n z / x)^{2} .
$$

Further polynomials $\psi_{m}(t)$, if needed, may be found expeditiously as follows. Define the polynomial $Q_{m}(t)$ by

$$
Q_{0}(t)=1, \quad Q_{1}(t)=5-t, \quad Q_{2}(t)=60-48 t+4 t^{2},
$$

and in general by the recurrence formula ${ }^{4}$

$$
Q_{m}=(6 m-(6 m-4) t) Q_{m-1}-4 t(1-t) Q_{m-1}^{\prime}+(1-t) \sum_{\lambda=2}^{m-1} Q_{\lambda-1} Q_{m-\lambda} .
$$

Then the $m$ th term $T_{m}$ of the asymptotic series occurring in (4) may be written

$$
T_{m}=\frac{1}{2}(-4 n)^{-m} \int_{2}^{\infty} Q_{m}\left(t^{2}\right) t^{-3 m-1} d t .
$$

In other words, if we denote the coefficient of $t^{k}$ in $Q_{m}(t)$ by $A_{k}(m)$ and define the polynomial $\phi_{m}(t)$ by

$$
\phi_{m}(t)=\sum_{k=0}^{m} A_{k}^{(m)} t^{t} /(3 m-2 k),
$$

then

$$
T_{m}=(-1)^{m} 2^{-2 m-1} n^{-m} z^{-3 m} \phi_{m}\left(z^{2}\right) .
$$

As a matter of fact, the polynomial

$$
\psi_{m}(t)=\phi_{m}(1+t)
$$

has simpler coefficients than $\phi_{m}(t)$ and since $\phi_{m}\left(z^{2}\right)=\psi_{m}\left(x^{2}\right)$ we have given $T_{m}$ partly in terms of $x$. In doing this we are following MEISSEL ${ }^{5}$ whose formulas for $J_{n}(n x)$ also involve $\psi_{m}(t)$ for $m \leqq 6$. A comparison of his extended results reveals four errors in his $\psi_{6}\left(\sec ^{2} \alpha\right)$. For denominators

$$
1,4,192,256,128,768 \text {, read } 1,4,48,16,8,48 .
$$

This incorrect polynomial has produced an error in the corresponding term in the formula as quoted by WATSON ${ }^{6}$

$$
\text { for 3072, 768, 41280, read 192, 48, } 2580 .
$$

Watson has another error (not due to MEISSEL) in the polynomial where 98720 is a misprint for 78720; noted by W. G. Bickley, Phil. Mag., s. 7, v. 34,1943 , p. 45 .

To illustrate the behavior of (4) for a moderate value of $x$ we take $x=\frac{3}{4}$ so that $z=\frac{5}{4}$. In this case (4) becomes:

$$
\begin{aligned}
\ln I_{n}(3 n / 4)=\left(\frac{5}{4}\right. & -\ln 3) n-\frac{1}{2} \ln n-\frac{1}{2} \ln (5 \pi / 2)-1 / 150 n-99 / 3125 n^{2} \\
& -1044097 / 175781250 n^{3}+868626 / 48828125 n^{4} \\
& +857735827 / 76904296875 n^{5} \\
& -4925585088 / 152587890625 n^{6} \\
& +4590654771247901 / 100135803222656250 n^{7} \\
& +278080398059472 / 2384185791015625 n^{8}+\cdots .
\end{aligned}
$$


For $n=100$ these various terms are, to 20 decimals,

\begin{tabular}{|c|c|c|}
\hline 15.13877 & 11331890 & \\
\hline 2.30258 & 5092994045 & 68402 \\
\hline 1.03051 & 0308884078 & 40331 \\
\hline .00006 & 6666666666 & 66667 \\
\hline .00000 & $31680<00000$ & 00000 \\
\hline .00000 & 0005939751 & 8222 \\
\hline .00000 & 0000177894 & 60480 \\
\hline+.00000 & 0000001115 & 32887 \\
\hline .00000 & 0000000032 & 28031 \\
\hline .00000 & 0000000000 & 45844 \\
\hline+.00000 & 000000000 & \\
\hline
\end{tabular}

and their sum is

$$
11.80560589088346549 \cdots \text {. }
$$

Thus

$$
I_{100}(75)=134001.4488018810 .
$$

D. H. L.

\footnotetext{
${ }^{1}$ Such Bessel functions have been used recently at the statistical laboratory of the University of California in preparation of certain statistical tables to appear in Annals of Math. Statistics. See also: J. WISHART, "A note on the distribution of the correlation ratio," Biometrika, v. 24, 1932, p. 454, formula (27).

2 The most extensive tables of $I_{n}(x)$ are in B.A.A.S., Math. Tables, v. 6, Bessel Functions, part I, Cambridge, 1937, Tables VI and VIII.

J. W. Nicholson, Phil. Mag. s. 6, v. 20, 1910, p. 938-943.

4 The $Q^{\prime}$ s may be checked by the relation $Q_{m}(1)=4^{m}$; also $m(m+1) \int_{0}^{1} t^{2 m-1} Q_{m}\left(t^{-2}\right) d t$ $=2^{2 m+1}\left|B_{m+1}\right|$, where $B_{k}$ is the $k$ th Bernoulli number, in the notation of Lucas.

D. F. E. Meissel, Astr. Nach. v. 130, 1892, cols. 363-4.

- G. N. WATSON, A Treatise on the Theory of Bessel Functions, Cambridge 1922, p. 228.
}

\section{Mathematical Tables in Phil. Mag.}

The important scientific periodical London, Edinburgh \& Dublin Philosophical Magazine and Journal of Science, has been published under different titles since 1798, in 7 series of volumes (varying in number from 11 to 68). In recent years the usual print abbreviation of the title of this periodical has been Phil. Mag. In what follows it is proposed to list, with a few added notes, mathematical tables in Phil. Mag., s. 3, v. 18 to s. 7, v. 34, no. 235, inclusive, 1841-1943. It will be observed that more than half of the Tables listed were published after the last world war started in 1914, a period of unparalleled scientific development. Many mathematical tables have been already published as the result of problems arising in the prosecution of the present war; the number is likely to be greatly increased in the next few years.

S. 3

1. G. B. AIRY "On diffraction of an annular aperture," v. 18, 1841, p. 7. Table of $E\left[=J_{0}(e)\right]=(1 / 2 \pi) \int_{0}^{2 \pi} \cos (e \cos \theta) d \theta$, and of $E^{2}$, for $e=[0.0(0.2) 10.0 ; 4 D]$. Airy had an earlier table of $2 J_{1}(e) / e$ of the same scope in Cambridge Phil. So., Trans., v. 5, 1835, p. 291. In B.A.A.S., Math. Tables, v. 6, Bessel Functions, Cambridge, 1937, $J_{0}$ is given, among other values, for $e=[0.0(0.2) 25.0 ; 8 D]$. The value of $J_{0}(40)$, to $7 D$, was computed by W. R. Hamilton, Phil: Mag., s. 4, v. 14, 1857, p. 381. 